\title{
Temporal expression patterns of fruit-specific $\alpha$ - EXPANSINS during cell expansion in bell pepper (Capsicum annuum L.)
}

\section{CURRENT STATUS: UNDER REVIEW}

BMC Plant Biology $\mathbf{B} M C$ Series

Savithri Nambeesan
University of Georgia
sunamb@uga.eduCorresponding Author

Andrés Mayorga-Gómez

University of Georgia

DOI:

10.21203/rs.3.rs-18016/v1

SUBJECT AREAS

Plant Molecular Biology and Genetics

KEYWORDS

cell wall loosening, gene expression, fruit growth and development, bell pepper 
Abstract

Background

Expansins (EXP) facilitate non-enzymatic cell wall loosening during several phases of plant growth and development including fruit growth, internode expansion, pollen tube growth, leaf and root development, and during abiotic stress responses. In this study, we characterized the spatial and temporal expression pattern of $C$. annuum $\alpha$ - EXPANSINS (CaEXPA) genes. Additionally, we correlated fruit-specific CaEXPA expression with the rate of cell expansion during bell pepper fruit development.

\section{Results}

Spatial expression patterns revealed that CaEXPA13 was up-regulated in vegetative tissues and flowers, with the most abundant expression in mature leaves. Expression of CaEXPA4 was associated with stems and roots. CaEXPA3 was expressed abundantly in flower at anthesis suggesting a role for CaEXPA3 in flower development. Temporal expression analysis revealed that 9 out of the 21 genes were highly expressed during fruit development. Of these, expression of six genes, CaEXPA5, CaEXPA7, CaEXPA12, CaEXPA14 CaEXPA17 and CaEXPA19 were abundant 7 to 21 days after anthesis (DAA), whereas CaEXP6 was strongly expressed between 14 and 28 DAA. Further, this study revealed that fruit growth and cell expansion occur throughout bell pepper development until ripening, with highest rates of fruit growth and cell expansion occurring between 7 and 14 DAA. The expression of CaEXPA14 and CaEXPA19 positively correlated with the rate of cell expansion, suggesting their role in post-mitotic cell expansion-mediated growth of the bell pepper fruit. In this study, we also identified high transcript abundance of CaEXPA9 during ripening.

\section{Conclusion}

This is the first genome-wide study of CaEXPA expression during fruit growth and development. We identified a fruit-specific EXPA that may be important in facilitating cell expansion during growth and cell wall loosening during ripening in bell pepper. These EXPA genes could be important targets for future manipulation of fruit size and ripening characteristics.

Full Text

Due to technical limitations, full-text HTML conversion of this manuscript could not be completed. 
However, the manuscript can be downloaded and accessed as a PDF.

Figures

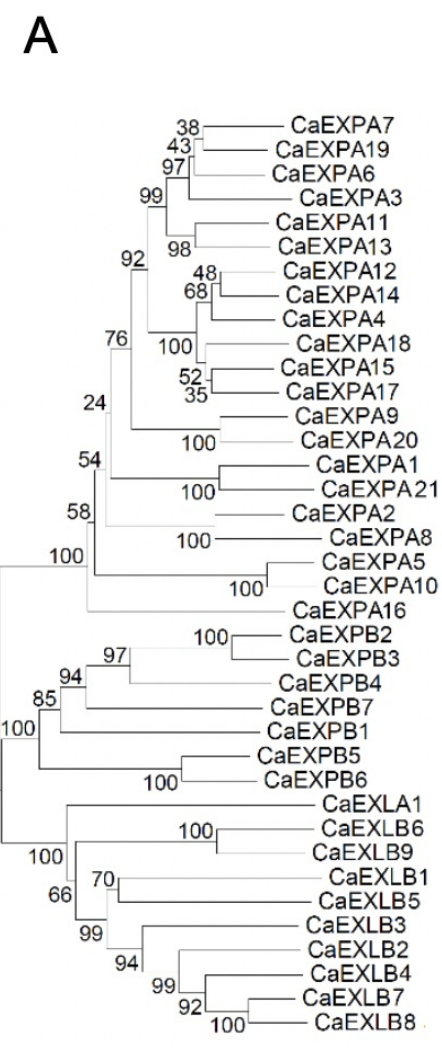

$\overline{0.05}$
B

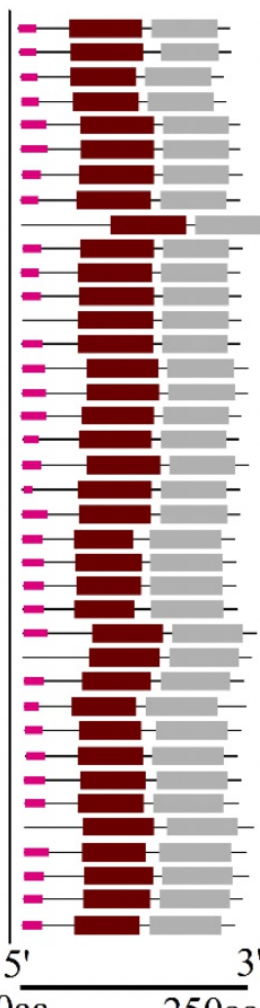

C

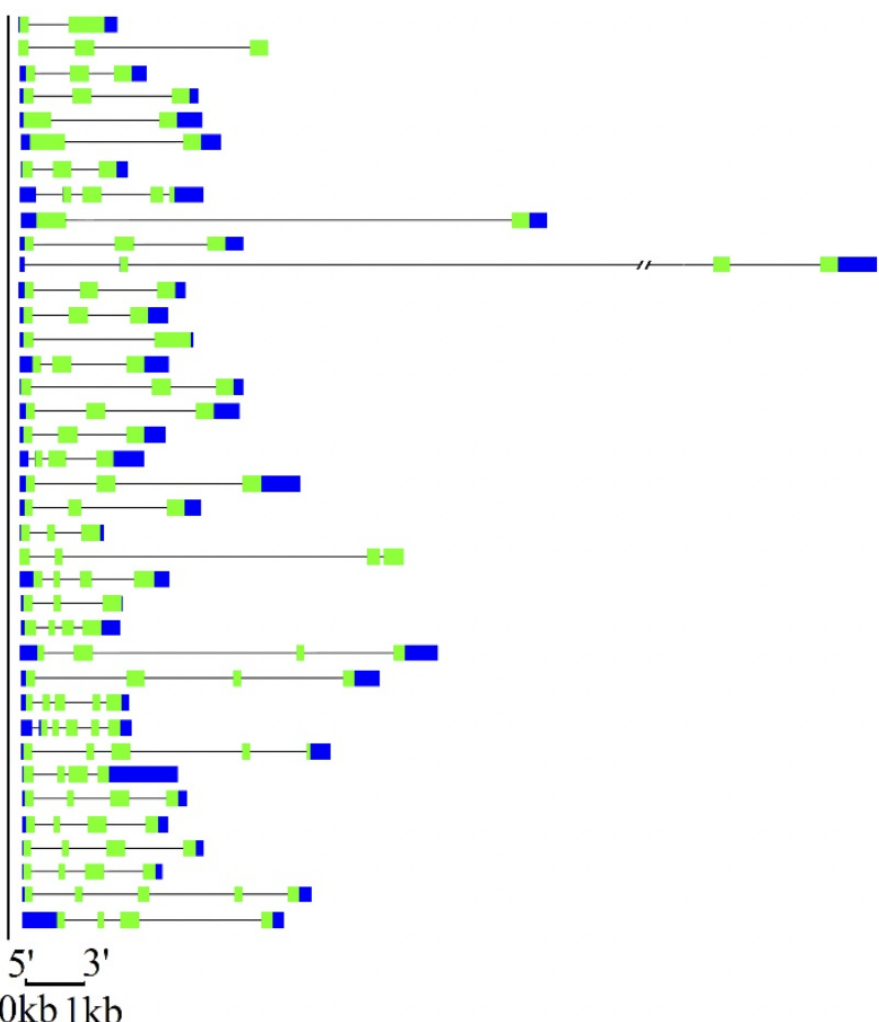

$\backsim$ Signal peptide $\square$ DPBB $₫$ Pollen allergen $₫$ Exon $\square 5^{\prime} / 3^{\prime}$ UTR - Intron $-1 / 500 \mathrm{bp}$

Figure 1

Phylogenetic analysis (A), schematic visualization of protein (B), and gene structure (C) of

EXPANSINS in C. annuum. A. Phylogenetic tree performed using the neighbor join method in MEGA 7.0 with 1000 bootstraps with p-distance model. B. Protein structure showing the signal peptide (pink), double-psi beta-barrel (DPBB; burgundy) and pollen allergen (grey) domains. Scale, 250 amino acids. C. Gene structure with exons in green, introns as a solid line and 5' and $3^{\prime}$ UTR in blue. Break in second intron of CaEXPA15 is 500 bp.

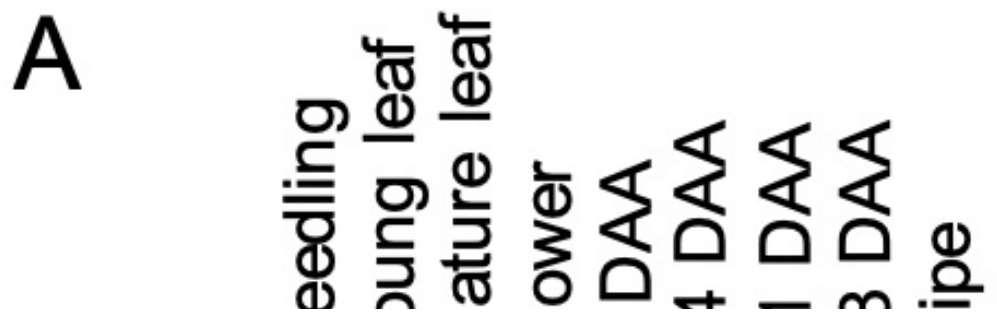




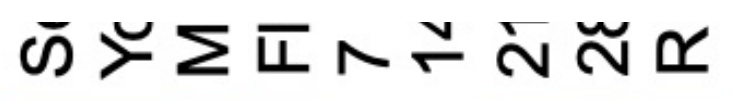

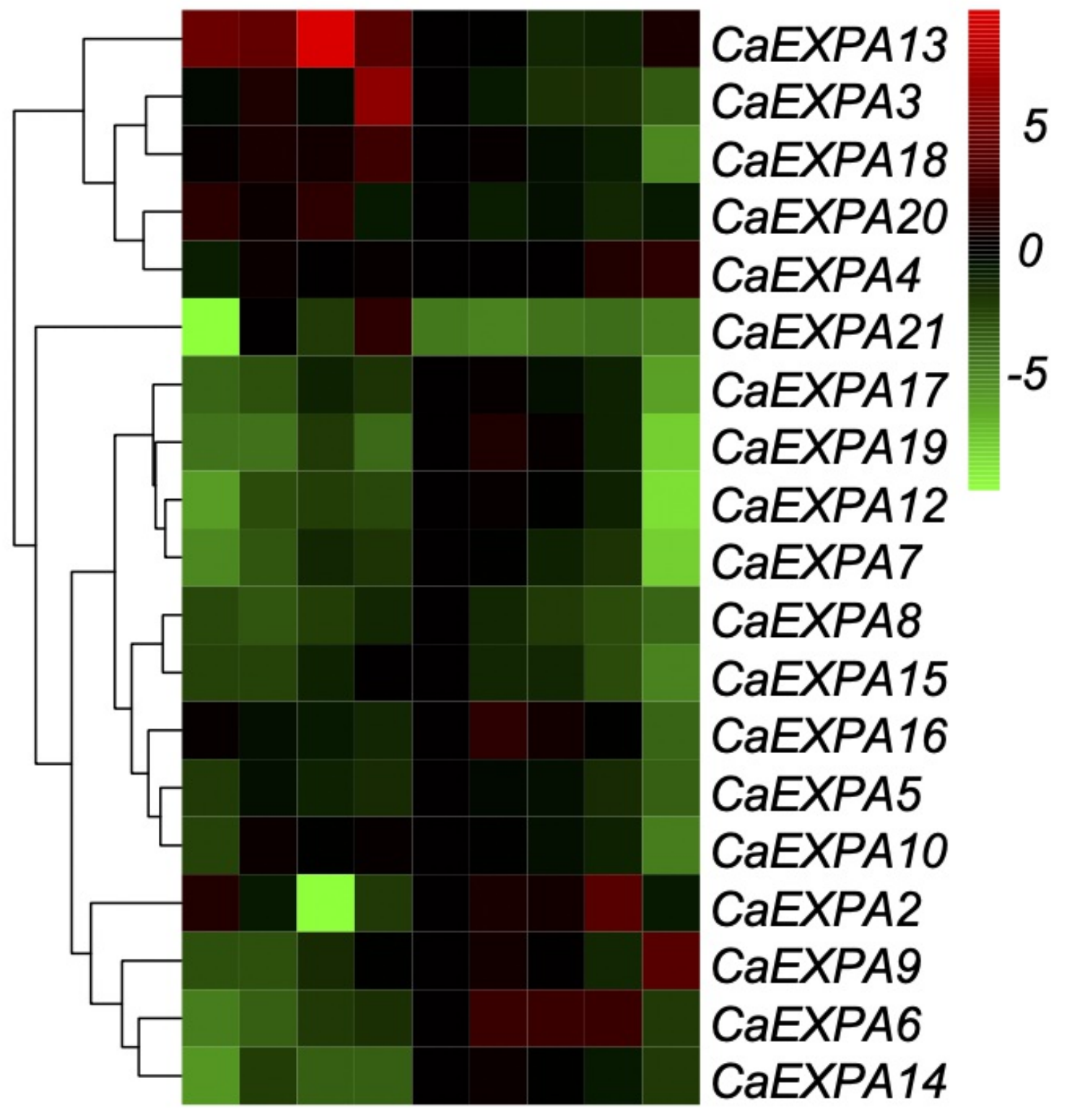

B

\section{总憘}

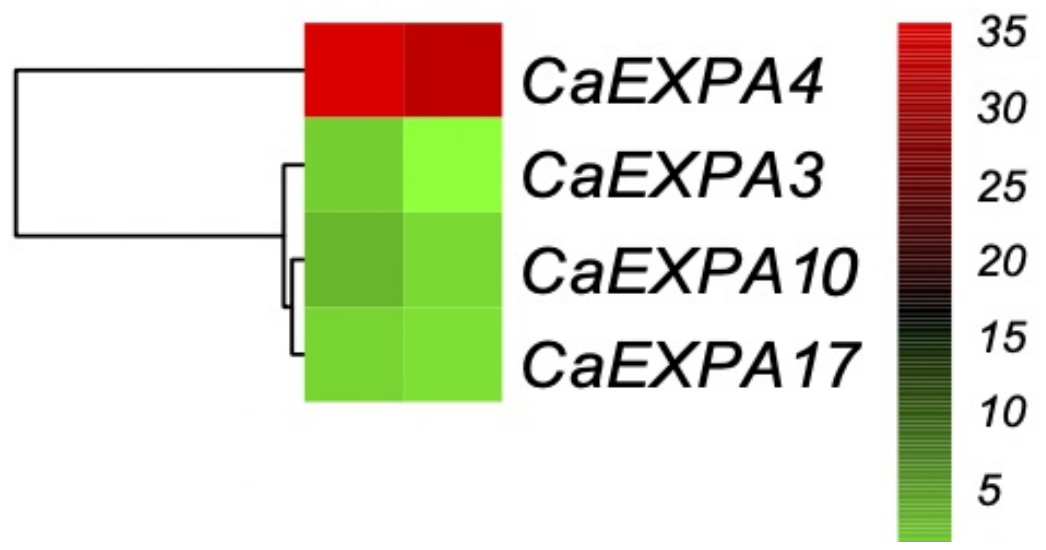


Figure 2

. Spatio-temporal expression patterns of CaEXPA. A. Transcript abundance of each CaEXPA gene in every tissue and fruit developmental time-point was normalized to its expression at 7 DAA, except CaEXPA21 normalized to young leaf. B. Expression patterns of CaEXPA in root and stem. Only four out of the 21 CaEXPA genes were expressed in root and stem tissue.

Transcript abundance of all 4 genes were normalized to that of CaEXPA10 in root. Tissues are displayed vertically and CaEXP genes horizontally. Color scale from green to red indicates higher and lower transcript abundance, respectively. Expression is presented in a Log2 scale.
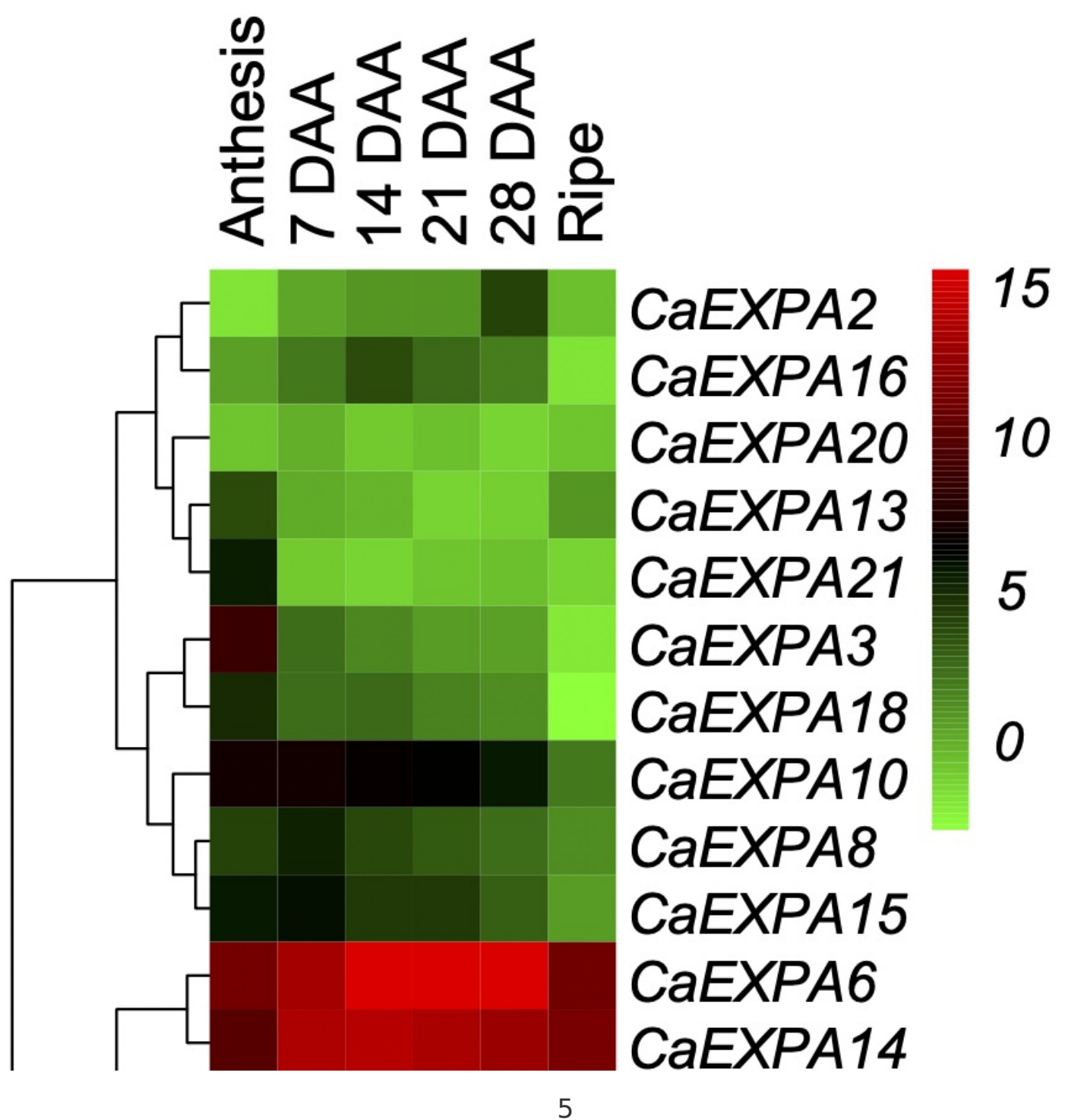


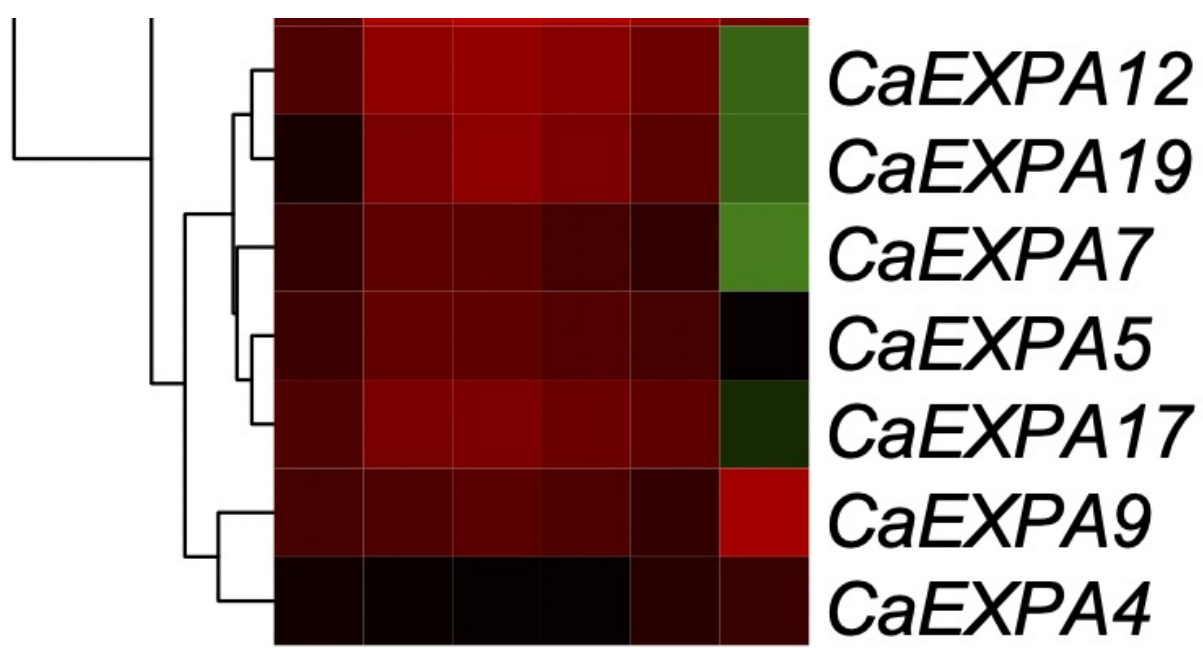

Figure 3

Temporal expression patterns of CaEXPA during flower and fruit development. Transcript abundance of all CaEXPA genes was normalized to that of CaEXP21 at 7 DAA. Tissues are displayed vertically and CaEXPA genes horizontally. Color scale from green to red indicates higher and lower transcript abundance, respectively. Expression is presented in a Log2 scale.
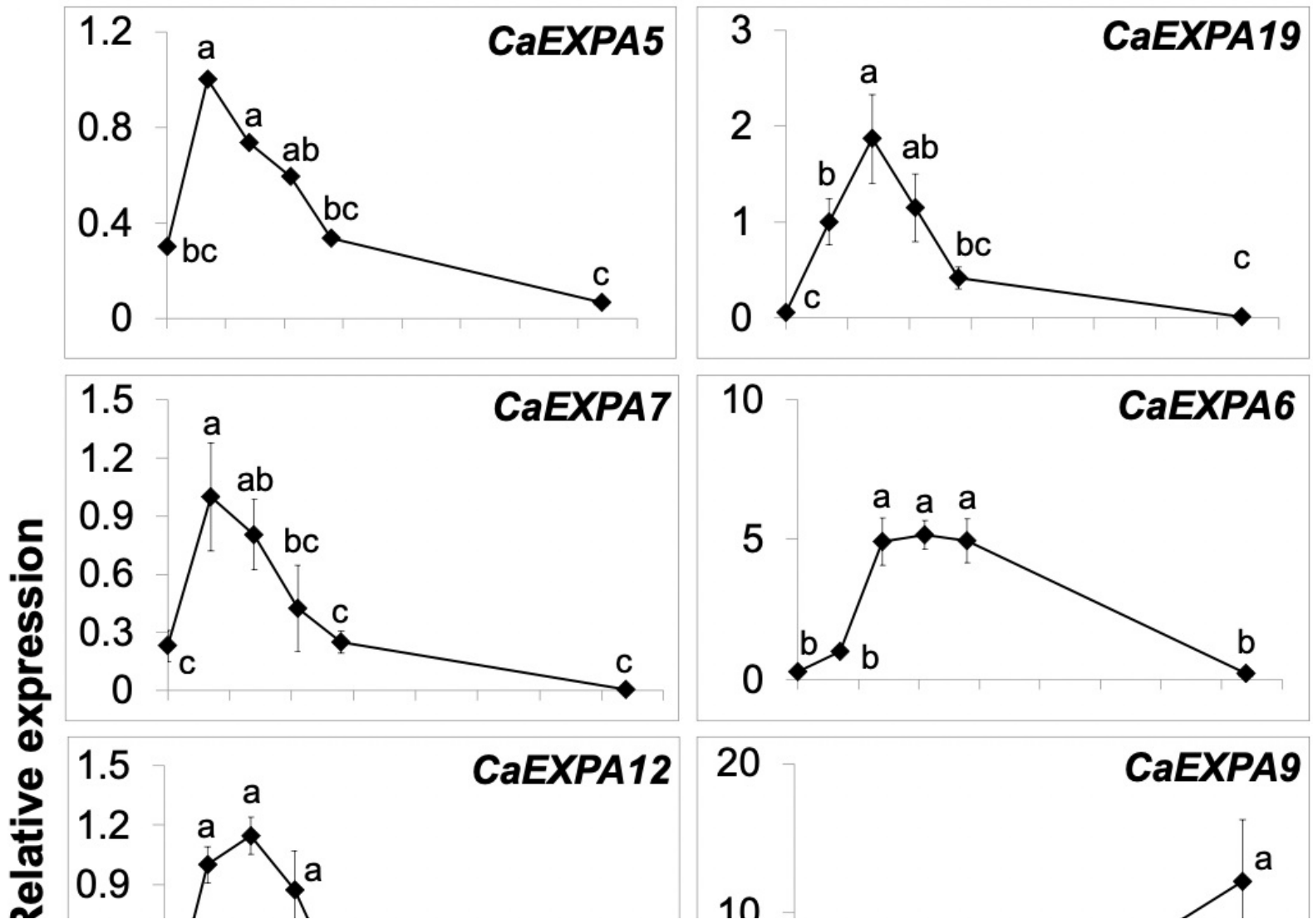

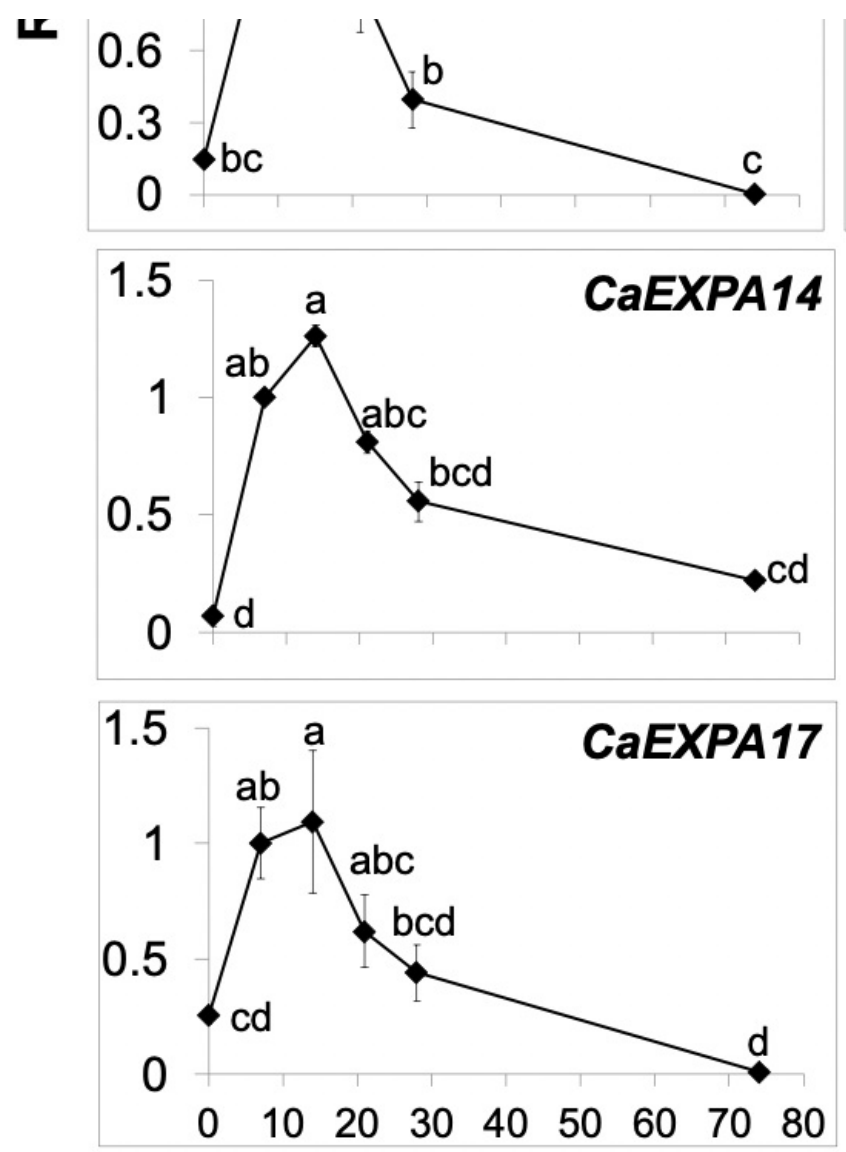
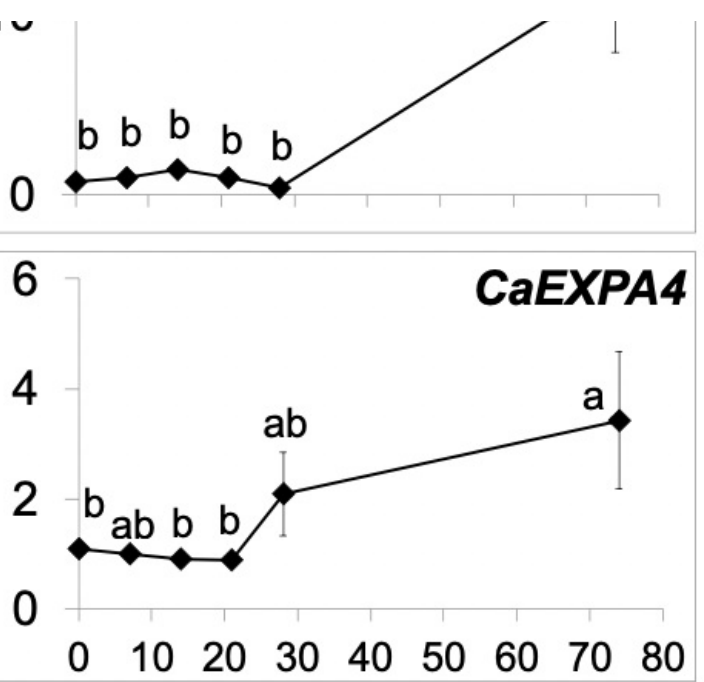

\section{Days after anthesis}

Figure 4

Relative transcript abundance of nine CaEXP genes in flower and fruit development. These nine CaEXP genes displayed relatively higher transcript abundance in Fig. 3. Values are means and standard errors of at least three replicates. Means separation was performed using Tukey's HSD following test of significance using ANOVA $(\alpha=0.05)$. Means followed by a different letter are significantly different. 

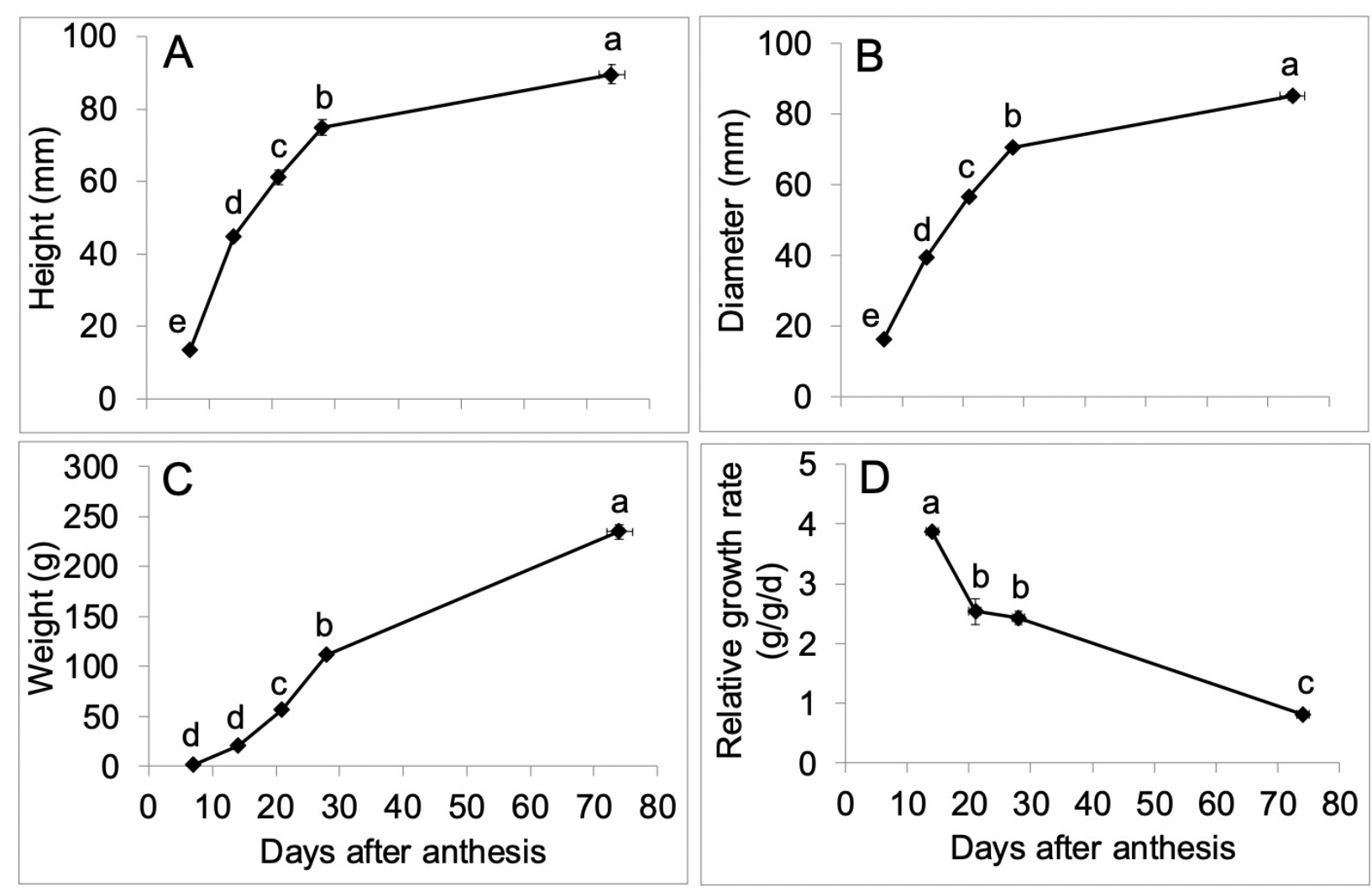

Figure 5

A. Fruit height, B. Fruit diameter, C. Fruit weight at five time-points during fruit development at various days after anthesis (DAA): 7, 14, 21, 28 DAA and ripening. D. Relative growth rate calculated using fruit weight $(\mathrm{g} / \mathrm{g} / \mathrm{d})$ at four different lapses of time; from 7-14 DAA, 14-21 DAA, 21-28 DAA and 28 DAA to ripening. Means separation was performed using Tukey's HSD following test of significance using ANOVA $(\alpha=0.05)$. Means followed by a different letter are significantly different. 


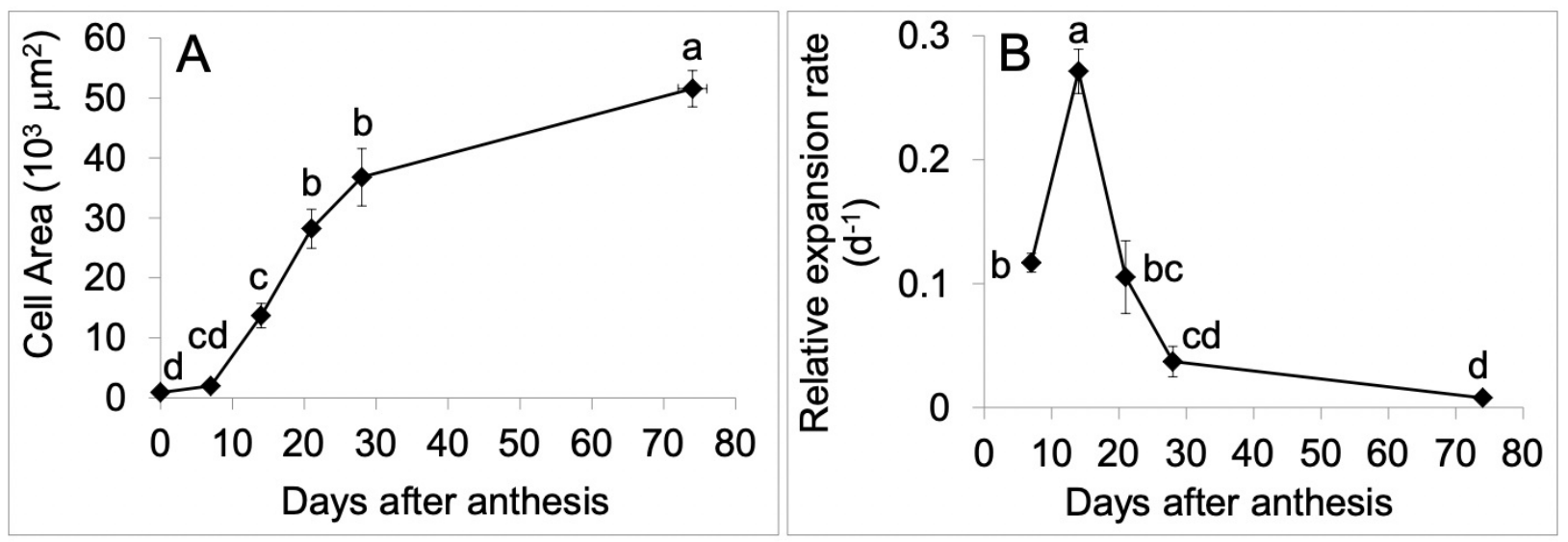

Figure 6

A. Fruit cell area (x $103 \mu \mathrm{m} 2)$ at various stages during fruit development: $0,7,14,21,28$

DAA and ripening. $B$. Relative fruit cell expansion rate (d-1) from 0-7 DAA, 7-14 DAA, 14-21

DAA, 21-28 DAA and 28 DAA to ripening. Means separation was performed using Tukey's HSD following test of significance using ANOVA $(\alpha=0.05)$. Means followed by a different letter are significantly different. 